\title{
Application of a light-front coupled cluster method
}

\author{
Sophia S. Chabysheva and John R. Hiller \\ Department of Physics \\ University of Minnesota-Duluth \\ Duluth, Minnesota 55812 \\ (Dated: December 17, 2018)
}

\begin{abstract}
As a test of the new light-front coupled-cluster method in a gauge theory, we apply it to the nonperturbative construction of the dressed-electron state in QED, for an arbitrary covariant gauge, and compute the electron's anomalous magnetic moment. The construction illustrates the spectator and Fock-sector independence of vertex and self-energy contributions and indicates resolution of the difficulties with uncanceled divergences that plague methods based on Fock-space truncation.
\end{abstract}

a Presented by S.S. Chabysheva at LIGHTCONE 2011, 23-27 May 2011, Dallas. To appear in the proceedings. 


\section{INTRODUCTION}

We wish to construct a nonperturbative solution for the dressed-electron state in QED, as an eigenstate of the light-front QED Hamiltonian, and use it to compute the electron's anomalous magnetic moment. Traditionally, such a calculation is done as a truncated expansion of the eigenstate in a Fock basis. As discussed elsewhere in these proceedings [1], truncation causes a number of difficulties but can be avoided with use of the new light-front coupled-cluster (LFCC) method [2]. Here we give details of an application of the method begun in [1]. Our notation for light-cone coordinates [3, 4] can also be found there.

The eigenvalue problem to solve is $\mathcal{P}^{-}|\psi\rangle=\frac{M^{2}+P_{\perp}^{2}}{P^{+}}|\psi\rangle$. The starting point of the LFCC method is to build the eigenstate as $|\psi\rangle=\sqrt{Z} e^{T}|\phi\rangle$ from a valence state $|\phi\rangle$ and an operator $T$ that increases particle number but conserves all relevant quantum numbers. This leads to an effective Hamiltonian $\overline{\mathcal{P}^{-}}=e^{-T} \mathcal{P}^{-} e^{T}$, which in practice is computed with use of its Baker-Hausdorff expansion, $\overline{\mathcal{P}^{-}}=\mathcal{P}^{-}+\left[\mathcal{P}^{-}, T\right]+\frac{1}{2}\left[\left[\mathcal{P}^{-}, T\right], T\right]+\cdots$. The new eigenvalue problem $\overline{\mathcal{P}^{-}}|\phi\rangle=\frac{M^{2}+P_{\perp}^{2}}{P^{+}}|\phi\rangle$ is projected onto the valence and orthogonal sectors

$$
P_{v} \overline{\mathcal{P}^{-}}|\phi\rangle=\frac{M^{2}+P_{\perp}^{2}}{P^{+}}|\phi\rangle, \quad\left(1-P_{v}\right) \overline{\mathcal{P}^{-}}|\phi\rangle=0 .
$$

To have a finite system of equations, the operator $T$ and the projection $1-P_{v}$ are truncated, with the latter chosen to provide as many equations as are needed to determine the functions in the truncated $T$. This automatically truncates the Baker-Hausdorff expansion of the effective Hamiltonian. What is not truncated is the exponentiation of $T$ that builds the eigenstate. Thus the eigenstate retains all Fock states consistent with the quantum numbers of the valence state.

The calculation of matrix elements requires some care to avoid infinite sums over the Fock basis. Consider the matrix element $\left\langle\psi_{2}|\hat{O}| \psi_{1}\right\rangle$ of an operator $\hat{O}$. Define $\left|\psi_{i}\right\rangle=\sqrt{Z_{i}} e^{T_{i}}\left|\phi_{i}\right\rangle$, with $Z_{i}=1 /\left\langle\phi_{i}\left|e^{T_{i}^{\dagger}} e^{T_{i}}\right| \phi_{i}\right\rangle$,

$$
\overline{O_{i}}=e^{-T_{i}} \hat{O}^{T_{i}}=\hat{O}_{i}+\left[\hat{O}_{i}, T\right]+\frac{1}{2}\left[\left[\hat{O}_{i}, T\right], T\right]+\cdots
$$

and, to avoid the infinite sum in the denominator,

$$
\left\langle\widetilde{\psi}_{i}\right|=\langle\phi| \frac{e^{T_{i}^{\dagger}} e_{i}^{T}}{\left\langle\phi\left|e^{T_{i}^{\dagger}} e^{T_{i}}\right| \phi\right\rangle}=Z_{i}\langle\phi| e^{T_{i}^{\dagger}} e^{T_{i}}=\sqrt{Z_{i}}\left\langle\psi_{i}\right| e^{T_{i}}
$$

We then have

$$
\left\langle\psi_{2}|\hat{O}| \psi_{1}\right\rangle=\sqrt{Z_{1} / Z_{2}}\left\langle\widetilde{\psi_{2}}\left|\overline{O_{2}} e^{-T_{2}} e^{T_{1}}\right| \phi_{1}\right\rangle=\sqrt{Z_{2} / Z_{1}}\left\langle\widetilde{\psi}_{1}\left|\overline{O_{1}^{\dagger}} e^{-T_{1}} e^{T_{2}}\right| \phi_{2}\right\rangle^{*} .
$$

Therefore, we can compute the matrix element as

$$
\left\langle\psi_{2}|\hat{O}| \psi_{1}\right\rangle=\sqrt{\left\langle\tilde{\psi}_{2}\left|\overline{O_{2}} e^{-T_{2}} e^{T_{1}}\right| \phi_{1}\right\rangle\left\langle\widetilde{\psi}_{1}\left|\overline{O_{1}^{\dagger}} e^{-T_{1}} e^{T_{2}}\right| \phi_{2}\right\rangle^{*}}
$$

In the diagonal case, this reduces to the form discussed in [1]:

$$
\langle\psi|\hat{O}| \psi\rangle=\langle\widetilde{\psi}|\bar{O}| \phi\rangle
$$




\section{APPLICATION TO QED}

As a test of the LFCC method, we consider the dressed-electron state truncated to exclude positrons but retaining an infinite number of photons. From this state, we compute the anomalous moment from a spin-flip matrix element of the current. The different spin states, for both the physical and PV eigenstates, are computed simultaneously with a single $T$ operator; the matrix element can then be computed from the simpler diagonal form.

\section{A. Effective Hamiltonian}

We use a QED Lagrangian regulated by Pauli-Villars (PV) fields [5]

$\mathcal{L}=\sum_{i=0}^{2}(-1)^{i}\left[-\frac{1}{4} F_{i}^{\mu \nu} F_{i, \mu \nu}+\frac{1}{2} \mu_{i}^{2} A_{i}^{\mu} A_{i \mu}-\frac{1}{2} \zeta\left(\partial^{\mu} A_{i \mu}\right)^{2}\right]+\sum_{i=0}^{2}(-1)^{i} \bar{\psi}_{i}\left(i \gamma^{\mu} \partial_{\mu}-m_{i}\right) \psi_{i}-e \bar{\psi} \gamma^{\mu} \psi A_{\mu}$

where the interaction is written in terms of null fields

$$
\psi=\sum_{i=0}^{2} \sqrt{\beta_{i}} \psi_{i}, \quad A_{\mu}=\sum_{i=0}^{2} \sqrt{\xi_{i}} A_{i \mu}, \quad F_{i \mu \nu}=\partial_{\mu} A_{i \nu}-\partial_{\nu} A_{i \mu}
$$

and the coupling coefficients are constrained by

$$
\xi_{0}=1, \quad \sum_{i=0}^{2}(-1)^{i} \xi_{i}=0, \quad \beta_{0}=1, \quad \sum_{i=0}^{2}(-1)^{i} \beta_{i}=0
$$

with a zero index for physical fields and nonzero for PV fields. Further, we require chiral symmetry restoration [6] and zero photon mass [7], to fix $\xi_{2}$ and $\beta_{2}$. The theory is quantized with a light-front analog of the Stueckelberg method [8]. The light-front Hamiltonian, without positron contributions, is [1, 5]

$$
\begin{aligned}
\mathcal{P}^{-}= & \sum_{i, s} \int d \underline{p} \frac{m_{i}^{2}+p_{\perp}^{2}}{p^{+}}(-1)^{i} b_{i, s}^{\dagger}(\underline{p}) b_{i, s}(\underline{p})+\sum_{l, \lambda} \int d \underline{k} \frac{\mu_{l \lambda}^{2}+k_{\perp}^{2}}{k^{+}}(-1)^{l} \epsilon^{\lambda} a_{l \lambda}^{\dagger}(\underline{k}) a_{l \lambda}(\underline{k}) \\
& +\sum_{i j l s \sigma \lambda} \int d y d \vec{k}_{\perp} \int \frac{d \underline{p}}{\sqrt{16 \pi^{3} p^{+}}}\left\{h_{i j l}^{\sigma s \lambda}\left(y, \vec{k}_{\perp}\right)\right. \\
& \left.\times a_{l \lambda}^{\dagger}\left(y p^{+}, y \vec{p}_{\perp}+\vec{k}_{\perp}\right) b_{j s}^{\dagger}\left((1-y) p^{+},(1-y) \vec{p}_{\perp}-\vec{k}_{\perp}\right) b_{i \sigma}(\underline{p})+\text { H.c. }\right\},
\end{aligned}
$$

with the $h_{i j l}^{\sigma s \lambda}$ being known functions and $\epsilon=(-1,1,1,1)$ the metric signature for the physical photon.

We truncate the $T$ operator to just simple photon emission from a fermion:

$$
T=\sum_{i j l s \sigma \lambda} \int d y d \vec{k}_{\perp} \int \frac{d \underline{p}}{\sqrt{16 \pi^{3}}} \sqrt{p^{+}} t_{i j l}^{\sigma s \lambda}\left(y, \vec{k}_{\perp}\right) a_{l \lambda}^{\dagger}\left(y p^{+}, y \vec{p}_{\perp}+\vec{k}_{\perp}\right) b_{j s}^{\dagger}\left((1-y) p^{+},(1-y) \vec{p}_{\perp}-\vec{k}_{\perp}\right) b_{i \sigma}(\underline{p}) \text {. }
$$



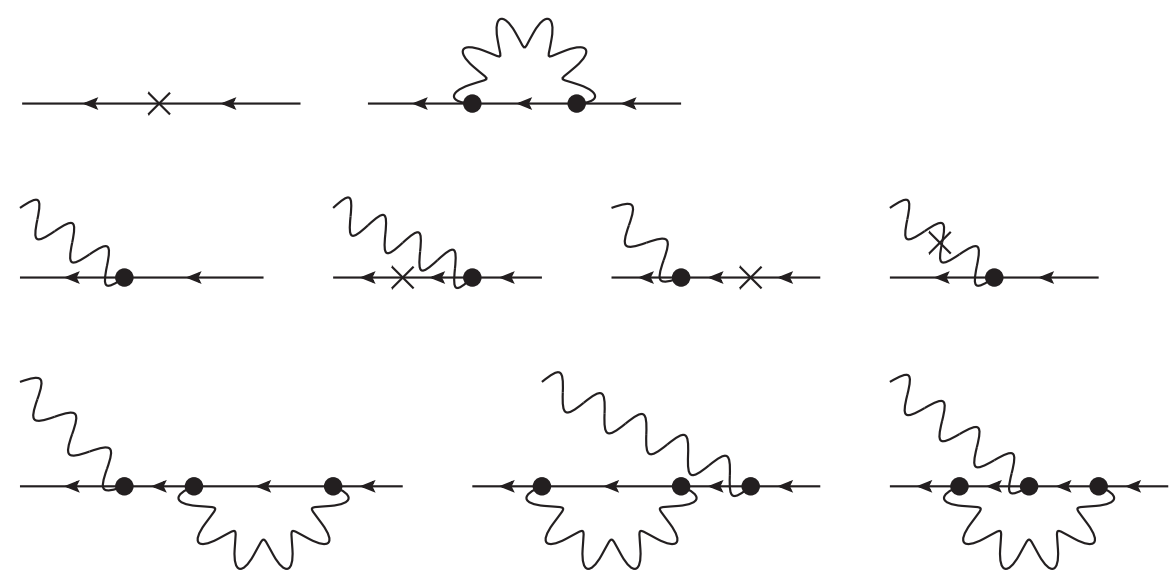

FIG. 1. Graphical representation of the terms of the effective Hamiltonian in Eq. (2.6) of the text. Each graph represents an operator that annihilates an electron and creates either a single electron or an electron and photon. The crosses indicate light-front kinetic-energy contributions.

The effective Hamiltonian, excluding terms that annihilate the valence state of a single electron, becomes

$$
\begin{aligned}
\overline{\mathcal{P}^{-}}= & \sum_{i j s} \int d \underline{p}(-1)^{i}\left[\delta_{i j} \frac{m_{i}^{2}+p_{\perp}^{2}}{p^{+}}+\frac{I_{j i}}{p^{+}}\right] b_{j, s}^{\dagger}(\underline{p}) b_{i, s}(\underline{p}) \\
& +\sum_{i j l s \sigma \lambda} \int d y d \vec{k}_{\perp} \int \frac{d \underline{p}}{\sqrt{16 \pi^{3} p^{+}}}\left\{h_{i j l}^{\sigma s \lambda}\left(y, \vec{k}_{\perp}\right)+\frac{1}{2} V_{i j l}^{\sigma s \lambda}\left(y, \vec{k}_{\perp}\right)\right. \\
& +\left[\frac{m_{j}^{2}+k_{\perp}^{2}}{1-y}+\frac{\mu_{l \lambda}^{2}+k_{\perp}^{2}}{y}-m_{i}^{2}\right] t_{i j l}^{\sigma s \lambda}\left(y, \vec{k}_{\perp}\right) \\
& \left.+\frac{1}{2} \sum_{i^{\prime}} \frac{I_{j i^{\prime}}}{1-y} t_{i i^{\prime} l}^{\sigma s \lambda}\left(y, \vec{k}_{\perp}\right)-\sum_{j^{\prime}}(-1)^{i+j^{\prime}} t_{j^{\prime} j l}^{\sigma s \lambda}\left(y, \vec{k}_{\perp}\right) I_{j^{\prime} i}\right\} \\
& \times a_{l \lambda}^{\dagger}\left(y p^{+}, y \vec{p}_{\perp}+\vec{k}_{\perp}\right) b_{j s}^{\dagger}\left((1-y) p^{+},(1-y) \vec{p}_{\perp}-\vec{k}_{\perp}\right) b_{i \sigma}(\underline{p}),
\end{aligned}
$$

with the self-energy contribution

$$
I_{j i}=(-1)^{i} \sum_{i^{\prime} l s \lambda}(-1)^{i^{\prime}+l} \epsilon^{\lambda} \int \frac{d y d k_{\perp}^{2}}{16 \pi^{3} p^{+}} h_{j i^{\prime} l}^{\sigma s \lambda *}\left(y, \vec{k}_{\perp}\right) t_{i i^{\prime} l}^{\sigma s \lambda}\left(y, \vec{k}_{\perp}\right),
$$

and the vertex loop correction

$$
\begin{aligned}
V_{i j l}^{\sigma s \lambda}\left(y, \vec{k}_{\perp}\right)= & \sum_{i^{\prime} j^{\prime} l^{\prime} s^{\prime} \sigma^{\prime} \lambda^{\prime}}(-1)^{i^{\prime}+l^{\prime}+j^{\prime}} \epsilon^{\lambda^{\prime}} \int \frac{d y^{\prime} d \vec{k}_{\perp}^{\prime}}{16 \pi^{3}} \frac{\theta\left(1-y-y^{\prime}\right)}{\left(1-y^{\prime}\right)^{1 / 2}(1-y)^{3 / 2}} \\
& \times h_{j j^{\prime} l^{\prime} l^{\prime} \lambda^{\prime} *}\left(\frac{y^{\prime}}{1-y}, \vec{k}_{\perp}^{\prime}+\frac{y^{\prime}}{1-y} \vec{k}_{\perp}\right) t_{i^{\prime} j^{\prime} l l}^{\sigma^{\prime} \prime^{\prime} \lambda}\left(\frac{y}{1-y^{\prime}}, \vec{k}_{\perp}+\frac{y}{1-y^{\prime}} \vec{k}_{\perp}^{\prime}\right) t_{i i^{\prime} l^{\prime}}^{\sigma \sigma^{\prime} \lambda^{\prime}}\left(y^{\prime}, \vec{k}_{\perp}^{\prime}\right)
\end{aligned}
$$

A graphical representation of the terms in $\overline{\mathcal{P}^{-}}$is given in Fig. 1. 


\section{B. Eigenvalue problems}

Because $\overline{\mathcal{P}^{-}}$is not Hermitian, we have both right and left-hand valence states

$$
\left|\phi^{ \pm}(\underline{P})\right\rangle=\sum_{i} z_{i} b_{i \pm}^{\dagger}(\underline{P})|0\rangle, \quad\left\langle\widetilde{\phi}^{ \pm}(\underline{P})\right|=\langle 0| \sum_{i} \tilde{z}_{i} b_{i \pm}(\underline{P}) .
$$

The projections of the right and left-hand eigenvalue problems onto the valence sector,

$$
P_{v} \overline{\mathcal{P}^{-}} P_{v}\left|\phi^{ \pm}(\underline{P})\right\rangle=\frac{M^{2}+P_{\perp}^{2}}{P^{+}}\left|\phi^{ \pm}(\underline{P})\right\rangle
$$

and

$$
\left(P_{v} \overline{\mathcal{P}^{-}} P_{v}\right)^{\dagger}\left|\widetilde{\phi}^{ \pm}(\underline{P})\right\rangle=\frac{M^{2}+P_{\perp}^{2}}{P^{+}}\left|\widetilde{\phi}^{ \pm}(\underline{P})\right\rangle,
$$

yield

$$
m_{i}^{2} z_{a i}^{ \pm}+\sum_{j} I_{i j} z_{a j}^{ \pm}=M_{a}^{2} z_{a i}^{ \pm}
$$

and

$$
m_{i}^{2} \tilde{z}_{a i}^{ \pm}+\sum_{j}(-1)^{i+j} I_{j i} \tilde{z}_{a j}^{ \pm}=M_{a}^{2} \tilde{z}_{a i}^{ \pm},
$$

with $a=0,1$ and $M_{a}$ the ath eigenmass. The valence eigenvectors are orthonormal and complete, in the following sense:

$$
\sum_{i}(-1)^{i} \tilde{z}_{a i}^{ \pm} z_{b i}^{ \pm}=(-1)^{a} \delta_{a b}, \quad \sum_{a}(-1)^{a} z_{i a}^{ \pm} \tilde{z}_{j a}^{ \pm}=(-1)^{i} \delta_{i j} .
$$

Projection of the right-hand eigenvalue problem onto $|e \gamma\rangle$, orthogonal to $|\phi\rangle$, gives

$$
\begin{aligned}
& \sum_{i}(-1)^{i} z_{a i}^{ \pm}\left\{h_{i j l}^{ \pm s \lambda}\left(y, \vec{k}_{\perp}\right)+\frac{1}{2} V_{i j l}^{ \pm s \lambda}\left(y, \vec{k}_{\perp}\right)+\left[\frac{m_{j}^{2}+k_{\perp}^{2}}{1-y}+\frac{\mu_{l \lambda}^{2}+k_{\perp}^{2}}{y}-m_{i}^{2}\right] t_{i j l}^{ \pm s \lambda}\left(y, \vec{k}_{\perp}(2 .\right.\right. \\
& \left.+\frac{1}{2} \sum_{i^{\prime}} \frac{I_{j i^{\prime}}}{1-y} t_{i i^{\prime} l}^{ \pm s \lambda}\left(y, \vec{k}_{\perp}\right)-\sum_{j^{\prime}}(-1)^{i+j^{\prime}} t_{j^{\prime} j l}^{ \pm s \lambda}\left(y, \vec{k}_{\perp}\right) I_{j^{\prime} i}\right\}=0 .
\end{aligned}
$$

To partially diagonalize in flavor, we define

$$
C_{a b l}^{ \pm s \lambda}\left(y, \vec{k}_{\perp}\right)=\sum_{i j}(-1)^{i+j} z_{a i}^{ \pm} z_{b j}^{ \pm} t_{i j l}^{ \pm s \lambda}\left(y, \vec{k}_{\perp}\right) .
$$

With analogous definitions for $H, I$, and $V$, we have

$$
\left[M_{a}^{2}-\frac{M_{b}^{2}+k_{\perp}^{2}}{1-y}-\frac{\mu_{l \lambda}^{2}+k_{\perp}^{2}}{y}\right] C_{a b l}^{ \pm s \lambda}\left(y, \vec{k}_{\perp}\right)=H_{a b l}^{ \pm s \lambda}\left(y, \vec{k}_{\perp}\right)+\frac{1}{2}\left[V_{a b l}^{ \pm s \lambda}\left(y, \vec{k}_{\perp}\right)-\sum_{b^{\prime}} \frac{I_{b b^{\prime}}}{1-y} C_{a b^{\prime} l}^{ \pm s \lambda}\left(y, \vec{k}_{\perp}\right)\right]
$$

to be solved simultaneously with the valence-sector equations, which depend on $C$ (or $t$ ) through the self-energy matrix $I$. Notice that the physical mass $M_{b}$ has replaced the bare mass in the kinetic energy term, without use of sector-dependent renormalization [9-12]. 
$\overline{\mathcal{P}}^{\dagger}$ :

The dual to the left-hand eigenstate $\left\langle\widetilde{\psi}^{ \pm}(\underline{P})\right|=\sqrt{Z}\left\langle\psi^{ \pm}(\underline{P})\right| e^{T}$ is a right eigenstate of

$$
\overline{\mathcal{P}}^{\dagger}\left|\tilde{\psi}^{ \pm}(\underline{P})\right\rangle=e^{T^{\dagger}} \mathcal{P}^{-} e^{-T^{\dagger}} \sqrt{Z} e^{T^{\dagger}}\left|\psi^{ \pm}(\underline{P})\right\rangle=\frac{M^{2}+P_{\perp}^{2}}{P^{+}}\left|\tilde{\psi}^{ \pm}(\underline{P})\right\rangle .
$$

It is normalized such that

$$
\left\langle\tilde{\psi}^{ \pm}\left(\underline{P^{\prime}}\right) \mid \phi(\underline{P})\right\rangle=\sqrt{Z}\left\langle\psi^{ \pm}\left(\underline{P^{\prime}}\right)\left|e^{T}\right| \phi^{ \pm}(\underline{P})\right\rangle=\left\langle\psi^{ \pm}\left(\underline{P^{\prime}}\right) \mid \psi(\underline{P})\right\rangle=\delta\left(\underline{P^{\prime}}-\underline{P}\right) .
$$

For the dressed electron, we construct this state as

$$
\left|\tilde{\psi}_{a}^{ \pm}(\underline{P})\right\rangle=\left|\tilde{\phi}_{a}^{ \pm}(\underline{P})\right\rangle+\sum_{j l s \lambda} \int d y d \vec{k}_{\perp} \sqrt{\frac{P^{+}}{16 \pi^{3}}} l_{a j l}^{ \pm s \lambda}\left(y, \vec{k}_{\perp}\right) a_{l \lambda}^{\dagger}\left(y P^{+}, y \vec{P}_{\perp}+\vec{k}_{\perp}\right) b_{j s}^{\dagger}\left((1-y) P^{+},(1-y) \vec{P}_{\perp}-\vec{k}_{\perp}\right)|0\rangle .
$$

We then flavor-diagonalize the left-hand wave functions

$$
D_{a b l}^{ \pm s \lambda}\left(y, \vec{k}_{\perp}\right) \equiv \sum_{j}(-1)^{j} z_{b j}^{s} l_{a j l}^{ \pm s \lambda}\left(y, \vec{k}_{\perp}\right)
$$

to obtain

$$
\left[M_{a}^{2}-\frac{M_{b}^{2}+k_{\perp}^{2}}{1-y}-\frac{\mu_{l \lambda}^{2}+k_{\perp}^{2}}{y}\right] D_{a b l}^{\sigma s \lambda}\left(y, \vec{k}_{\perp}\right)=\tilde{H}_{a b l}^{\sigma s \lambda}\left(y, \vec{k}_{\perp}\right)+W_{a b l}^{\sigma s \lambda}\left(y, \vec{k}_{\perp}\right)-\sum_{b^{\prime}} J_{b^{\prime} a}^{\sigma} \tilde{H}_{b^{\prime} b l}^{\sigma s \lambda}\left(y, \vec{k}_{\perp}\right),
$$

where

$$
\widetilde{H}_{a b l}^{\sigma s \lambda}\left(y, \vec{k}_{\perp}\right)=\sum_{i j}(-1)^{i+j} \tilde{z}_{a i} z_{b j} h_{i j l}^{\sigma s \lambda}\left(y, \vec{k}_{\perp}\right),
$$

$W_{a b l}^{\sigma s \lambda}$ is a vertex-correction analog of $V_{a b l}^{\sigma s \lambda}$, though linear in $D$, and $J_{b a}^{\sigma}$ is a self-energy analog of $I_{b a}$. Solutions for $M_{a}, z_{a i}^{\sigma}, \tilde{z}_{a i}^{\sigma}$, and $C_{a b l}^{\sigma s \lambda}$ are used as input.

\section{Anomalous moment}

We compute the anomalous moment $a_{e}$ from the spin-flip matrix element of the current $J^{+}=\bar{\psi} \gamma^{+} \psi$ [13], coupled to a photon of momentum $q$ in the Drell-Yan $\left(q^{+}=0\right)$ frame [14]:

$$
16 \pi^{3}\left\langle\psi_{a}^{\sigma}(\underline{P}+\underline{q})\left|J^{+}(0)\right| \psi_{a}^{ \pm}(\underline{P})\right\rangle=2 \delta_{\sigma \pm} F_{1}\left(q^{2}\right) \pm \frac{q^{1} \pm i q^{2}}{M_{a}} \delta_{\sigma \mp} F_{2}\left(q^{2}\right) .
$$

In limit of infinite PV masses, and with $M_{0}=m_{e}$ the electron mass, we have

$$
\begin{aligned}
F_{1}\left(q^{2}\right)=\frac{1}{\mathcal{N}}\left[1+\sum_{s} \int \frac{d y d \vec{k}_{\perp}}{16 \pi^{3}}\right. & \left\{\sum_{\lambda=1}^{2} l_{000}^{ \pm s \lambda *}\left(y, \vec{k}_{\perp}-y \vec{q}_{\perp}\right) t_{000}^{ \pm s \lambda}\left(y, \vec{k}_{\perp}\right)\right. \\
& \left.\left.-\sum_{\lambda=0}^{3} \epsilon^{\lambda} l_{000}^{ \pm s \lambda *}\left(y, \vec{k}_{\perp}\right) t_{000}^{ \pm s \lambda}\left(y, \vec{k}_{\perp}\right)\right\}\right]
\end{aligned}
$$

and

$$
F_{2}\left(q^{2}\right)= \pm \frac{2 m_{e}}{q^{1} \pm i q^{2}} \sum_{s} \sum_{\lambda=1}^{2} \int \frac{d y d \vec{k}_{\perp}}{16 \pi^{3}} l_{000}^{\mp s \lambda *}\left(y, \vec{k}_{\perp}-y \vec{q}_{\perp}\right) t_{000}^{ \pm s \lambda}\left(y, \vec{k}_{\perp}\right) / \mathcal{N}
$$


with

$$
\mathcal{N}=1-\sum_{s} \sum_{\lambda=0,3} \epsilon^{\lambda} \int \frac{d y d \vec{k}_{\perp}}{16 \pi^{3}} l_{000}^{ \pm s \lambda *}\left(y, \vec{k}_{\perp}\right) t_{000}^{ \pm s \lambda}\left(y, \vec{k}_{\perp}\right)
$$

A second term is absent in $F_{2}$ because $l$ and $t$ are orthogonal for opposite spins.

The $q^{2} \rightarrow 0$ limit can be taken, to find $F_{1}(0)=1$ and

$$
a_{e}=F_{2}(0)= \pm \frac{m_{e}}{\mathcal{N}} \sum_{s} \sum_{\lambda=1,2} \epsilon^{\lambda} \int \frac{d y d \vec{k}_{\perp}}{16 \pi^{3}} y l_{000}^{\mp s \lambda *}\left(y, \vec{k}_{\perp}\right)\left(\frac{\partial}{\partial k^{1}} \mp i \frac{\partial}{\partial k^{2}}\right) t_{000}^{ \pm s \lambda}\left(y, \vec{k}_{\perp}\right) .
$$

As a check, we can consider a perturbative solution

$$
t_{000}^{\sigma s \lambda}=l_{000}^{\sigma s \lambda}=h_{000}^{\sigma s \lambda} /\left[m_{e}^{2}-\frac{m_{e}^{2}+k_{\perp}^{2}}{1-y}-\frac{\mu_{l \lambda}^{2}+k_{\perp}^{2}}{y}\right] .
$$

Substitution into the expression for $a_{e}$ gives immediately the Schwinger result [15] of $\alpha / 2 \pi$, in the limit of zero photon mass, for any covariant gauge. A complete calculation requires a numerical solution of the eigenvalue problems for the left and right-hand wave functions.

\section{SUMMARY}

The LFCC method provides the means to solve for eigenstates of light-front Hamiltonians without truncation of Fock space and includes techniques for computing physical observables from matrix elements. The illustration given here shows how the method is applied to a calculation of the dressed-electron eigenstate of QED, including determination of the anomalous moment. A first-order perturbative solution yields the standard Schwinger term. A complete solution requires numerical techniques; work on this is in progress. The analysis and calculation is systematically improvable through the addition of terms to the exponentiated operator $T$. In particular, for the dressed electron, a term that adds electron-positron loops to the calculation can be included. A perturbative solution would then match ordinary perturbation theory to order $\alpha^{2}$; the nonperturbative solution provides a partial resummation of contributions from higher orders.

\section{ACKNOWLEDGMENTS}

This work was supported in part by the US Department of Energy through Contract No. DE-FG02-98ER41087 and by the Minnesota Supercomputing Institute through grants of computing time.

[1] Hiller, J.R., Chabysheva, S.S.: A light-front coupled cluster method. Contributed to these proceedings. To appear in Few-Body Systems.

[2] Chabysheva, S.S., Hiller, J.R.: A light-front coupled-cluster method for the nonperturbative solution of quantum field theories. arXiv:1103.0037 hep-ph] (2011)

[3] Dirac, P.A.M.: Forms of relativistic dynamics. Rev. Mod. Phys. 21, 392-399 (1949) 
[4] For reviews of light-cone quantization, see Burkardt, M.: Light front quantization. Adv. Nucl. Phys. 23, 1-74 (2002); Brodsky, S.J., Pauli, H.-C., Pinsky, S.S.: Quantum chromodynamics and other field theories on the light cone. Phys. Rep. 301, 299-486 (1998)

[5] Chabysheva, S.S., Hiller, J.R.: A first nonperturbative calculation in light-front QED for an arbitrary covariant gauge. Phys. Rev. D 84, 034001 (2011)

[6] Chabysheva, S.S., Hiller, J.R.: Restoration of the chiral limit in Pauli-Villars-regulated lightfront QED, Phys. Rev. D 79, 114017 (2009)

[7] Chabysheva, S.S., Hiller, J.R.: Nonperturbative Pauli-Villars regularizaton of vacuum polarization in light-front QED, Phys. Rev. D 82, 034004 (2010)

[8] Stueckelberg, E.C.G.: Interaction energy in electrodynamics and in the field theory of nuclear forces. Helv. Phys. Acta 11, 225-244 (1938); Coester, F.: Quantum electrodynamics with nonvanishing photon mass. Phys. Rev. 83, 798-800 (1951); Itzykson, C., Zuber, Z.-B.: Quantum Field Theory. McGraw-Hill, New York (1980)

[9] Perry, R.J., Harindranath, A., Wilson, K.G.: Light front Tamm-Dancoff field theory. Phys. Rev. Lett. 65, 2959-2962 (1990); Perry, R.J., Harindranath, A.: Renormalization in the light front Tamm-Dancoff approach to field theory. Phys. Rev. D 43, 4051-4073 (1991)

[10] Hiller, J.R., Brodsky, S.J.: Nonperturbative renormalization and the electron's anomalous moment in large- $\alpha$ QED. Phys. Rev. D 59, 016006 (1998)

[11] Karmanov, V.A., Mathiot, J.F., Smirnov, A.V.: Systematic renormalization scheme in lightfront dynamics with Fock space truncation. Phys. Rev. D 77, 085028 (2008); Nonperturbative calculation of the anomalous magnetic moment in the Yukawa model within truncated Fock space. Phys. Rev. D 82, 056010 (2010)

[12] Chabysheva, S.S., Hiller, J.R.: On the nonperturbative solution of Pauli-Villars regulated light-front QED: A comparison of the sector-dependent and standard parameterizations, Ann. Phys. 325, 2435-2450 (2010)

[13] Brodsky, S.J., Drell, S.D.: The anomalous magnetic moment and limits on fermion substructure. Phys. Rev. D 22, 2236-2243 (1980)

[14] Drell, S.D., Levy, D.J., Yan, T.M.: A field-theoretic model for electron-nucleon deep inelastic scattering. Phys. Rev. Lett. 22, 744-749 (1969)

[15] Schwinger, J.: On quantum electrodynamics and the magnetic moment of the electron. Phys. Rev. 73, 416-417 (1948); Quantum electrodynamics III: The electromagnetic properties of the electronradiative corrections to scattering. Phys. Rev. 76, 790-817 (1949) 